\title{
Morphomics Predicts Response to Ipilimumab in Patients With Stage IV Melanoma
}

\author{
MICHAEL S. SABEL, MD, ${ }^{1}$ JAY LEE, MD, ${ }^{1}$ ANRAN WANG, ${ }^{1}$ CHRISTOPHER LAO, MD, MPH, ${ }^{2}$ \\ SVEN HOLCOMBE, MS, ${ }^{1}$ AND STEWART WANG, MD, PhD ${ }^{1}$ \\ ${ }^{1}$ Department of Surgery, University of Michigan Health System, Ann Arbor, Michigan \\ ${ }^{2}$ Department of Medicine, University of Michigan Health System, Ann Arbor, Michigan
}

\begin{abstract}
Introduction: Factors predictive of response to immunotherapy are needed to select appropriate patients. As morphometric analysis can be an objective surrogate for underlying physiology, we explored the possibility that morphomics may predict response among stage IV melanoma patients treated with ipilimumab.

Methods: We identified stage IV melanoma patients treated with ipilimumab who had an appropriate CT scan within a 6 month window. Using semi-automated algorithms, we acquired several morphomic measurements. Toxicity and response rate compared by quartile using Fisher's exact test or chi-square, while survival after initiation of ipilimumab was compared by quartile using the log-rank test.

Results: While there was a significant correlation between toxicity and response $(P<.003)$, morphomics failed to predict either severity of toxicity or specific side effects. Psoas density was significantly associated with response rate, both excluding stable disease (36.4\% vs $9.1 \%, P=.054)$, and including stable disease $(54.5 \%$ versus $18.2 \%, P=0.045)$. Survival after initiation of ipilimumab was significantly associated with psoas density $(P=0.04)$ and visceral fat distance $(P=0.022)$.

Discussion: In an exploratory study of patients with metastatic melanoma being treated with ipilimumab, psoas density and spine-fascia distance correlated with response and survival. Pre-treatment morphomic analysis, as a correlate of underlying physiology, may help predict response to immunotherapy.

J. Surg. Oncol. 2015;112:333-337. (C) 2015 Wiley Periodicals, Inc.
\end{abstract}

KeY WoRDs: melanoma; ipilimumab; immunotherapy; morphomics

\section{INTRODUCTION}

Ipilimumab is a monoclonal antibody against cytotoxic $\mathrm{T}$ lymphocyte-associated antigen-4 (CTLA-4) approved for the treatment of unresectable or metastatic melanoma. Expressed on activated T-cells, CLTA-4 binds to B7 molecules on antigen presenting cells and serves to dampen specific T-cell responses. By blocking CTLA-4 binding, the negative signal is interrupted, resulting in a more robust $\mathrm{T}$-cell response. Both extended follow-up of phase II trials and a randomized phase III trial have established that ipilimumab can induce durable, potentially curative, tumor regression and improve overall survival [1,2].

As anti-CTLA- 4 blockade works non-specifically through the immune system, as opposed to directly targeting the tumor, there are no tumorspecific predictive factors that identify patients more or less likely to respond to therapy. Without this selection process, patients inherently unlikely to respond must be exposed to months of therapy, and the resultant toxicity, until the futility is realized. We previously described our experience with analytic morphomics in patients with stage III melanoma, demonstrating a strong correlation with survival, even after factoring for tumor characteristics, suggesting the biology of the host may be as important as the biology of the tumor [3]. As a surrogate for underlying physiology, morphomics may identify patients less capable of generating a clinically significant immune response, which may not only be prognostic, but predictive of response to biologic therapies. To explore this hypothesis, we performed an exploratory study of analytic morphomics among stage IV melanoma patients treated with ipilimumab.

\section{METHODS}

We conducted an IRB-approved search for patients treated with ipilimumab for metastatic melanoma at the University of Michigan. We next identified those patients who had CT scans of the abdomen and pelvis with IV contrast within a 6 month window of receiving their first dose of ipilimumab. CT scans were processed using semi-automated algorithms programmed into MATLAB v13.0 as described in previous work [4,5]. These algorithms use novel, high-throughput techniques to identify the linea alba and the anterior abdominal skin along the midline at each vertebral level from T12 to L4. The average distance between the linea alba and the anterior skin along T-12 to L4 was labeled the subcutaneous fat distance (SFD), and the average distance between the anterior aspect of the vertebra and the linea alba was labeled the visceral anterior-to posterior (AP) distance (VF). The sum of the SFD and visceral AP distance was labeled the total AP distance, or total body fat (TBF).

Both psoas area (PA) and psoas density (PD) were determined in our study population. Cross-sectional areas of the left and right psoas muscles at the level of the fourth lumber vertebra (L4) were measured. The area of the resulting enclosed regions was then computed to generate the cross-sectional area of the psoas muscles. Fatty infiltration of the psoas muscle was assessed by measuring the density, in Hounsfield Units (HU), within these regions, with lower HU reflecting more fat infiltration [6]. This highly reproducible method correlates with muscle triglyceride content on muscle biopsy [7-9].

*Correspondence to: Michael S. Sabel, MD, 3302 Cancer Center, 1500 East Medical Center Drive, Ann Arbor, MI 48109.

Fax: 734-647-9647. E-mail: msabel@umich.edu

Received 19 April 2015; Accepted 27 July 2015

DOI 10.1002/jso.24003

Published online 7 August 2015 in Wiley Online Library (wileyonlinelibrary.com). 
The data were classified categorically by tabulating the degree of response, the length of treatment, and individual side effects. Patients who had interval increase in number or size of lesions following treatment, deemed progression, were classified as no response; those who had both progression and regression were classified as mixed response; those with no change in disease were classified as stable response; those who had only regression in lesions were classified as positive response. Each individual side effect was noted in its own categorical variable, as well as the need for high-dose steroids or the need for therapy hold. Complication severity was further graded under mild, intermediate, or severe.

Statistical analysis of the data was done using SAS 9.2 and SPSS 22 software. Patient disease characteristics and the occurrences of toxicity from ipilimumab were compared using two-sample t- tests or analysis of variance techniques when the number of groups exceeded two. Linear regression models were used to assess the association between continuous covariates. Multivariate models were generated comparing the relationship between side effects and response. To evaluate the relationship between morphometric measures, toxicity from ipilimumab, response to ipilimumab, and overall survival, patients were stratified into quartiles based on the morphometric measures of interest. The response rates and occurrences of ipilimumab toxicity in each quartile were then compared using Fisher's exact test or chi-square test when the number of groups exceeded two. For overall survival, time was calculated from the date of initiation of ipilimumab until disease recurrence or death, or death, respectively. Patients not experiencing the endpoint of interest were censored on the date of their last known clinical follow-up. The Kaplan-Meier method was used to estimate survival probability in each quartile. Survival in the different quartiles was compared using the log-rank test. For all statistical tests, P-values at or below 0.05 were considered significant.

\section{RESULTS}

We identified 133 patients treated with ipimilumab for metastatic melanoma. Among this population, 48 had CT scans of the abdomen and pelvis with IV contrast, within a 6 month window of receiving their first dose of ipilimumab that could be processed and programmed into MATLAB v13.0. Patient and tumor characteristics for both sets of patients are presented in Table I.

\section{Toxicity}

We sought to examine whether toxicity from ipilimumab correlated with both response and with morphomics. The side effects of treatment were assessable in 129 patients ( 2 patients died before assessment and in 2 patients it was not possible to differentiate the side effects of treatment versus those of disease burden). The severity of complications related to ipilimumab treatment was categorized as none, mild, moderate and severe. Among these 129 patients, $36(28 \%)$ tolerated ipilimumab therapy without complication while 51 (39\%) had only mild complications. Moderate complications were seen in $24(17 \%)$ patients, and severe complications were seen in $17(13 \%)$.

TABLE I. Patient and Tumor Characteristics of Study Population

\begin{tabular}{|c|c|c|}
\hline & All patients $(n=133)$ & Patients with evaluable CT scans $(n=48)$ \\
\hline \multicolumn{3}{|l|}{ Gender } \\
\hline Male & $84(63 \%)$ & $32(66 \%)$ \\
\hline Female & $49(37 \%)$ & $16(34 \%)$ \\
\hline \multicolumn{3}{|l|}{ Age at melanoma diagnosis } \\
\hline Mean (Range) & $55.1(15-90)$ & $56.7(15-90)$ \\
\hline \multicolumn{3}{|l|}{ Primary histology } \\
\hline Nodular & $29(22 \%)$ & $12(25 \%)$ \\
\hline Superficial Spreading & $28(21 \%)$ & $8(16 \%)$ \\
\hline Occult Primary & $17(13 \%)$ & $7(14 \%)$ \\
\hline Ocular/Conjunctival & $13(10 \%)$ & $4(8 \%)$ \\
\hline Mucosal/Sinonasal & $6(4 \%)$ & $4(8 \%)$ \\
\hline Acral lentiginous & $6(4 \%)$ & $2(4 \%)$ \\
\hline Other/Unknown & $34(26 \%)$ & $11(23 \%)$ \\
\hline \multicolumn{3}{|c|}{ Primary Breslow Thickness (mm) } \\
\hline Mean (range) & $3.70(0.35-27)$ & $4.0(0.7-27)$ \\
\hline \multicolumn{3}{|l|}{ Primary Nodal Status } \\
\hline Negative & $27(20 \%)$ & $4(8 \%)$ \\
\hline Positive & $67(50 \%)$ & $25(52 \%)$ \\
\hline Not Staged/Unknown & $39(30 \%)$ & $19(40 \%)$ \\
\hline \multicolumn{3}{|l|}{ Prior Adjuvant Therapy } \\
\hline None & $108(80 \%)$ & $35(72 \%)$ \\
\hline Interferon & $15(11 \%)$ & $7(14 \%)$ \\
\hline Clinical Trial & $10(9 \%)$ & $2(4 \%)$ \\
\hline \multicolumn{3}{|l|}{ Age at stage IV diagnosis } \\
\hline Mean (Range) & $58.02(21-90)$ & $58.97(31-90)$ \\
\hline \multicolumn{3}{|c|}{ Time from Initial Diagnosis to Stage IV Disease } \\
\hline Simultaneous & $24(18 \%)$ & $14(29 \%)$ \\
\hline$<1$ year & $24(18 \%)$ & $8(16 \%)$ \\
\hline $1-2$ years & $29(22 \%)$ & $5(10 \%)$ \\
\hline $2-3$ years & $19(14 \%)$ & $9(18 \%)$ \\
\hline $3-5$ years & $14(10 \%)$ & $5(10 \%)$ \\
\hline$>5$ years & $23(18 \%)$ & $7(15 \%)$ \\
\hline \multicolumn{3}{|l|}{ 1st Line of Stage IV Treatment } \\
\hline Ipilimumab & $92(69 \%)$ & $32(66 \%)$ \\
\hline Vemurafenib & $8(6 \%)$ & $1(2 \%)$ \\
\hline High-dose IL-2 & $15(11 \%)$ & $9(18 \%)$ \\
\hline Dasatinib & $2(1 \%)$ & $0(0 \%)$ \\
\hline Temozolomide & $5(4 \%)$ & $2(4 \%)$ \\
\hline Other clinical trial agent & $11(6 \%)$ & $4(8 \%)$ \\
\hline
\end{tabular}


Response was categorized as none, mixed or partial response, stable disease or a positive response. Overall, there was a statistically significant correlation between the presence of complications and increasing response efficacy $(P=.0026)$. There was also a positive statistical significance between response and complication grade $(P=.0168)$, with response corresponding to severe complications when controlling for mild and intermediate complications. We were unable to demonstrate a correlation between any specific toxicity and outcome.

We examined whether psoas density, BMI and visceral abdominal girth predicted either the severity of side effects of ipilimumab or were associated with specific toxicities. The distribution of toxicity (none/ $\mathrm{mild} /$ moderate/severe) among the 48 patients with evaluable CT scans was similar to the entire group, with no complications in $20 \%$, mild in $45 \%$, moderate in $16 \%$ and severe complications in $18 \%$. We also examined specific toxicities including colitis, endocrinopathies, pruritis, nausea or fatigue. We were unable to demonstrate any statistically significant correlation between psoas density and the severity of ipilimumab side effects. There was no association between colitis, pruritis or nausea and psoas density. Endocrine side effects and fatigue were both more common among patients with lower psoas density ( $4 \%$ vs. $13 \%$ and $9 \%$ vs. $22 \%$, respectively), however these failed to reach statistical significance. There was no correlation between BMI and either severity of toxicity or specific side effects.

\section{Response and Outcome}

Of the 44 patients had complete analytic morphometry data and complete outcome data, higher psoas density was associated with improved response to ipilimumab. Specifically, patients in the highest quartile of psoas density (patients with the least fatty infiltration of the psoas muscle) had a response rate of $36.4 \%$, which was markedly higher than the response rate of $9.1 \%$ in the remaining patients $(P=0.054)$. When patients with stable disease were also included in the response rate, patients in the largest quartile of psoas density had a response rate of $54.5 \%$, which was significantly higher than the response rate of
$18.2 \%$ in the remaining patients $(P=0.045)$. Total psoas area, abdominal girth, and BMI were not significantly correlated with response to ipilimumab.

We also examined the relationship between morphometric measures and overall survival from the date of initiation of ipilimumab treatment. Patients were first stratified into quartiles based on the morphometric measures of interest. The Kaplan-Meier method was used to estimate survival probability in each quartile. There was a clear trend towards improved survival with patients stratified into quartiles based on psoas density, although this did not reach statistical significance. $(P=0.073)$. Comparing the top quartile against all other patients, there was a significantly improved survival compared to all other patients $(P=0.04)$, as shown in Figure 1. 1-year survival was $71.4 \%$ for patients in the largest quartile of psoas density, compared to $40.1 \%$ for all other patients.

Visceral fat distance was also significantly correlated with survival. Figure 2 shows survival with patient stratified into quartiles based on visceral fat distance. Patients in the largest quartile of visceral fat distance had worse survival compared to all other patients $(P=0.022)$. 1 -year survival for patients in the largest quartile of visceral fat distance was $25.0 \%$, compared to $56.3 \%$ for all other patients. Of note, there was no significant correlation between survival and total psoas area or BMI.

\section{DISCUSSION}

The relative benefit of systemic therapies, either in the adjuvant or metastatic setting, is often assessed by examining prognostic and predictive factors, the former meant to estimate the potential benefit of therapy and the latter meant to estimate the likelihood of response. In the past few years, we have seen the introduction of several new systemic agents in the treatment of melanoma; small molecule inhibitors for patients with mutations in the gene encoding BRAF, a key component of the mitogen-activated protein kinase signaling pathway, and immune checkpoint regulators. The likelihood of response to targeted therapies can be predicted by the presence of specific mutations in the BRAF gene. While a mutation in BRAF is not a guarantee of a positive

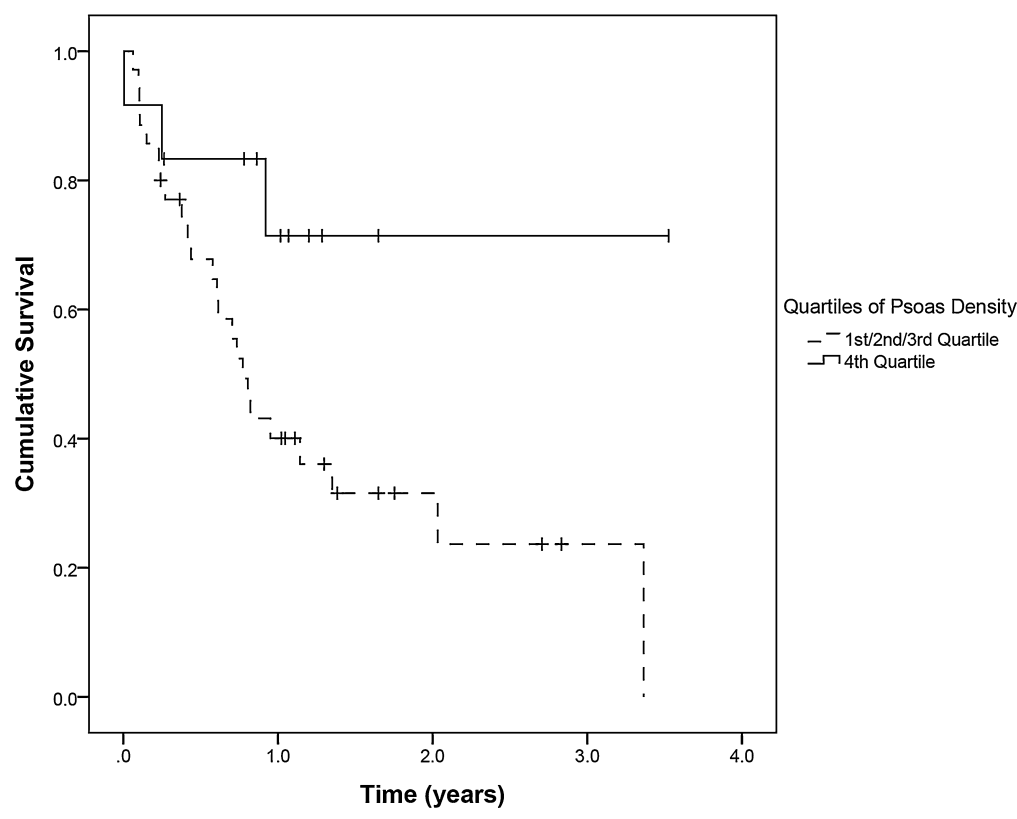

Figure 1. Cumulative Survival Stratified by Psoas Density. Patients with the most psoas density (least fatty infiltration) have a significantly better outcome after treatment with ipilimumab compared with patients with increasing fatty infiltration. 


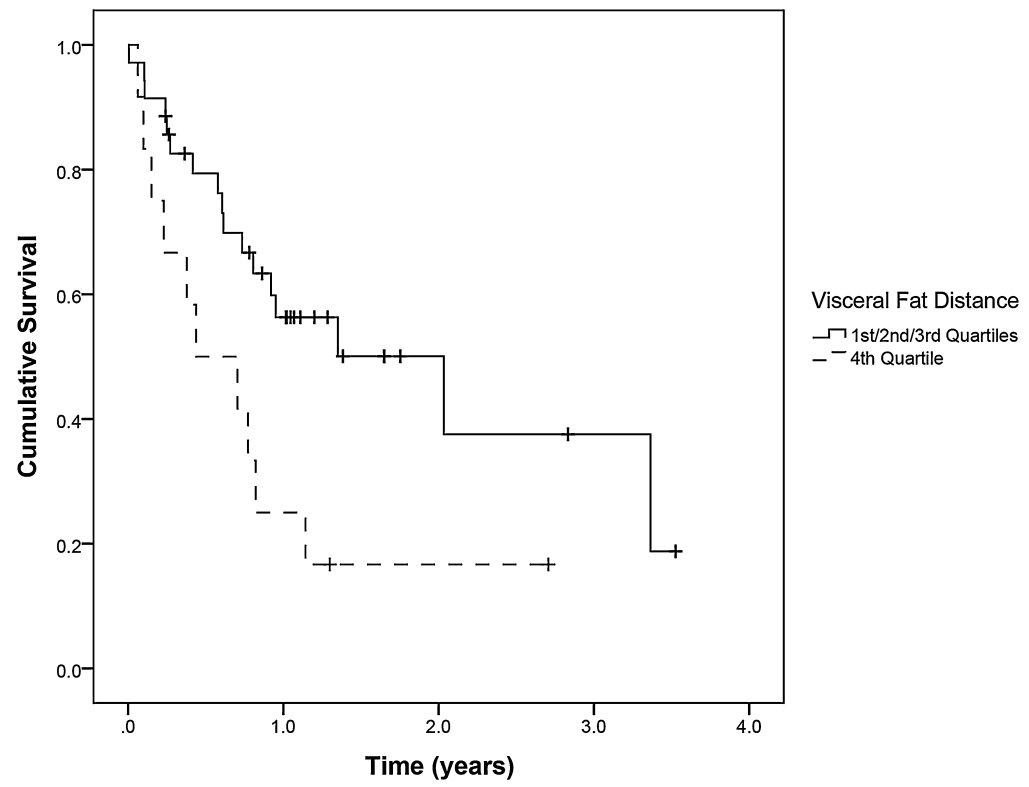

Figure 2. Cumulative Survival Stratified by Spine-Fascia Distance. Patients with the highest spine-fascia distance (largest visceral fat distance) have a significantly worse outcome after treatment with ipilimumab compared with patients with less visceral fat.

response to treatment, patients with wild-type B-RAF would not be expected to respond and are spared the morbidity of treatment.

There have been several attempts to identify biomarkers that may predict response to immunotherapies. This is particularly important so that these costly new treatments, which often have severe adverse reactions, can be targeted to those patients most likely to benefit. To date, there has been no surrogate or predictive marker for response to ipilimumab identified [10]. Absolute lymphocyte count (ALC) has been identified as a potential predictive marker, not just the ALC prior to therapy, but more importantly the change in ALC after two ipilimumab treatments $[11,12]$. Other reported potential markers include c-reactive protein (CRP), circulating regulatory T-cells (Treg), myeloid derived suppressor cells (MDSC), CD ${ }^{+}$ICOS $^{\text {high }}$ T-cells, expression of ICOS, or antibodies to the cancer testis antigen NY-ESO-1 [10,13-15]. However most of these need to be measured before and during therapy, meaning patients need to start and maintain therapy, already being exposed to some cost and potential toxicity, before one can assess whether they are likely to respond.

Morphomic analysis allows clinicians to map out the threedimensional anatomy and density of specific muscles, fat components and bone. This objective quantification may give a better assessment of the patients underlying physiology, and relative frailty, and morphomic analysis has evolved as a superb method for assessing risk in preoperative patients [4,5]. These morphologic variations may also predict toxicity and response to systemic therapy [16,17], and in situations where patient physiology and tumor biology interact, analytic morphomics may play be a significant prognostic factor. When examining patients with stage III melanoma, psoas density, a measure of the fatty infiltration of the psoas muscle, was significantly correlated with survival, even after factoring for age and known prognostic factors [3]. In renal cell carcinoma, body composition assessment has also been associated with prognosis [18].

Non-specific immunotherapies, where the patient is not given an immunologically active agent (such as with the administration of antibodies or adoptive T-cell therapy), but rather an agent that augments a pre-existing but clinically insufficient tumor-specific immune response, is based on the assumption that not only does that response exist, but can be augmented. It has long been known that aging is associated with impaired functionality of the immune system, but more recently, data has emerged linking body composition, nutrition, diet and frailty with alterations in the adaptive immune response $[19,20]$. And while age may be associated with changes in morphomics, there are multiple factors at play, and physiologic age can be quite different from chronologic age. For this reason, morphomics may give a better estimate of underlying functional status than age alone [21]. In the study of sarcopenia among stage III melanoma patients, when sarcopenia was included in multivariate analysis, age (long known to be a prognostic factor in melanoma) was no longer significant [3].

The results of this exploratory study do suggest that among patients with metastatic melanoma being treated with ipilimumab, both the response rate and survival were significantly associated with psoas density. Patients with the least fatty infiltration of the psoas muscle had a significantly higher response rate $(36.4 \%$ versus $9.1 \%, P=.05)$ and a longer cumulative survival from the initiation of ipilimumab treatment $(P=.04)$. Another morphometric measurement, spine-fascia distance, a measure of visceral fat, was also associated with survival. Patients with the greatest spine-fascia distance had a significantly worse survival $(P=.02)$. There are limitations to this study, in particular the small number of patients in our cohort who had CT scans of the abdomen and pelvis performed at the time they started ipilimumab treatment. Patients staged with PET/CT scans could not be analyzed as the CT scan is noncontrast and psoas density would not be comparable.

Despite these limitations, the results of this exploratory study are encouraging. There are several potential advantages to the use of morphomics in prediction of response to immunotherapy. The morphomic measurements described (psoas density, spine-fascia distance) can be obtained with relative ease from any CT scan of the abdomen and pelvis using oral and intravenous contrast, a test which is often ordered in the routine evaluation of the patient with stage IV melanoma. Therefore there would be minimal increased cost. In addition, as the test is obtained prior to the initiation of therapy, patients unlikely to response would be spared the cost and toxicity of initiating treatment before being able to determine the likelihood of success. These results strongly warrant further study with larger numbers in 
order to perform multivariate analysis, as well as a more objective delineation of what degree of psoas density (or visceral fat) might predict poor response to ipilimumab.

\section{REFERENCES}

1. Hodi FS,O'Day SJ, McDermott DF, et al.: Improved survival with ipilimubab in patients with metastatic melanoma. NEJM 2010;363:711-723.

2. Prieto PA, Yang JC, Sherry RM, et al.: CTLA-4 blockade with ipilimumab: Long-term follow-up of 177 patients with metastatic melanoma. Clin Cancer Res 2012;18:2039-2047.

3. Sabel MS, Lee J, Cai S, et al.: Sarcopenia as a prognostic factor among patients with stage III melanoma. Ann Surg Oncol 2011;18:3579-3585.

4. Englesbe MJ, Patel SP, He K, et al.: Sarcopenia and mortality after liver transplantation. J Am Coll Surg 2010;211:271-278.

5. Lee JS, He K, Harbaugh CM, et al.: Frailty, core muscle size, and mortality in patients undergoing open abdominal aortic aneurysm repair. J Vasc Surg 2011;53:912-917.

6. Baumgartner RN, Wayne SJ, Waters DL, et al.: Sarcopenic obesity predicts instrumental activities of daily living disability in the elderly. Obes Res 2004;12:1995-2004.

7. Villareal DT, Banks M, Sinacore DR, et al.: Effect of weight loss and exercise on frailty in obese, older adults. Arch Intern Med 2006;166:860-866.

8. Goodpaster BH, Kelley DE, Thaete FL, et al.: Skeletal muscle attenuation determined by computed tomography is associated with skeletal muscle lipid content. J Appl Physiol 2000;89:104-110.

9. Goodpaster BH, Thaete FL, Kelley DE: Skeletal muscle composition evaluated with computed tomography. Ann NY Acad Sci 2000;904:18-24.

10. Ascierto PA, Kalos M, Schaer DA, et al.: Biomarkers for immunostimulatory monocolonal antibodies in combination strategies for melanoma and other tumor types. Clin Cancer Res 2013;19:1009-1020.

11. Ku GY, Yuan J, Page DB, et al.: Single-institution experience with ipilimumab in advanced melanoma patients in the compassionate use setting: Lymphocyte count after 2 doses correlates with survival. Cancer 2010;116:1767-1775.

12. Berman D, Wolchock JD, weber J, et al.: Association of peripheral blood absolute lymphocyte count (ALC) and clinical activity in patients with advacned melanoma treated wtih ipilimumab (abstract). J Clin Oncol 2009;27:abstract3020.

13. Yuan J, Adamow M, Ginsberg BA, et al.: Integrated NY-ESO-1 antibody and CD8 + T-cell responses correlate wtih clinical benefit in advanced melanoma patients treated with ipilimumab. Proc Natl Acad Sci USA 2011;108:16723-16728.

14. Fu T, He Q, Sharma P: The ICOS/ICOSL pathway is required for optimal antitumor responses mediated by anti-CTLA-4 therapy. Cancer Res 2011;16:3485-3494.

15. Kitano S, Postow MA, Cortez C, et al.: Myeloid derived supressor cell quantity prior to treatment with ipilimumab at $10 \mathrm{mg} / \mathrm{kg}$ to predict for overall survival in patients with metastatic melanoma (abstract). J Clin Oncol 2012;30:abstract2518.

16. Antoun S, Baracos VE, Birdsell L, et al.: Low body mass index and sarcopenia associatedw tih dose-limiting toxicity of sorafenib in patients with renal cell carcinoma. Ann Oncol 2010;21: $1594-1598$.

17. Prado CM, Baracos VE, McCargar LJ, et al.: Sarcopenia as a determinant of chemotherapy toxicity and time to tumor progression in metastatic breast cancer patients receving capecitabine treatment. Clin Cancer Res 2009;15:2920-2926.

18. Antoun S, Lanoy E, Albiges-Sauvin L, et al.: Clinical implications of body composition assessment by computed tomography in metastatic renal cell carcinoma. Expert Rev Anticancer Ther 2014;14:279-288.

19. Yao X, Li H, Leng SX: Inflammation and immune system alterations in frailty. Clin Geriatr Med 2011;27:79-87.

20. Offord EA, Karagounis LG, Vidal K, et al.: Nutrition and the biology of human aging: bone health \& osteoporosis/ sarcopenia/immune deficiency. J Nutr Health Aging 2013;17: 712-716.

21. Miller AS, Min LC, Diehl KM, et al.: Analytic morphomics coresponds to functional status in older patients. J Surg Res 2014;192:19-26. 